\title{
Different contexts change the impression of animacy
}

\author{
Giulia Parovel $^{1} \cdot$ Stefano Guidi $^{1} \cdot$ Karina Kreß $^{1}$
}

Published online: 27 November 2017

(C) The Psychonomic Society, Inc. 2017

\begin{abstract}
The aim of this research was to explore the effect of different spatiotemporal contexts on the perceptual saliency of animacy, and the extent of the relationship between animacy and other related properties such as emotions and intentionality. Paired-comparisons and ratings were used to compare the impressions of animacy elicited by a small square moving on the screen, either alone or in the context of a second square. The context element was either static or moving showing an animate-like or a physical-like trajectory, and the target object moved either toward it or away from it. The movement of the target could also include animacy cues (caterpillar-like expanding/contracting phases). To determine the effect of different contexts on the emergence of emotions and intentions, we also recorded and analysed the phenomenological reports of participants. The results show that the context significantly influences the perception of animacy, which is stronger in dynamic contexts than in static ones, and also when the target is moving away from the context element than when it is approaching it. The free reports reveal different proportions in emotional or intentional attributions in the different conditions: in particular, the "moving away" condition is related to negative emotions, while the "approaching" condition evokes positive emotions. Overall, the results suggest that animacy is a graded concept that can be articulated in more general characteristics, like simple aliveness, and more specific ones, like
\end{abstract}

Electronic supplementary material The online version of this article (https://doi.org/10.3758/s13414-017-1439-x) contains supplementary material, which is available to authorized users.

Giulia Parovel

giulia.parovel@unisi.it

1 Department of Social, Political and Cognitive Sciences, University of Siena, Via Roma, 56, 53100 Siena, Italy intentions or emotions, and that the spatiotemporal contingencies of the context play a crucial role in making them evident.

Keywords Visual perception · Animacy · Intentionality · Causal perception $\cdot$ Negativity bias $\cdot$ Experimental phenomenology

\section{Introduction}

Since Heider and Simmel's pioneering demonstration of 1944, it had been acknowledged that simple geometrical shapes, despite their inanimate character, can express aliveness, emotions and intentional attributes by virtue of their motion.

The impression of aliveness and meaningfulness of these events is surprisingly vivid, immediate and compelling (see Scholl \& Tremoulet, 2000 and Scholl \& Gao, 2013, for reviews), and many studies demonstrate they are strongly related to low-level visual processing, despite the seemingly higher-level impressions they prompt. According to several authors, we perceive animacy and intentionality automatically, in the same manner that we perceive spatial depth or optical-geometrical illusions (Scholl \& Gao, 2013).

The emergence of animacy in moving shapes has been often related to minimal kinematic cues like self-propelling motion in the absence of an external cause, thus implying the perceptual evidence of a hidden energy source (Premack, 1990; Dasser, Ulbaek, \& Premack, 1989; Markson \& Spelke, 2006; Csibra, 2008), and other violations of the conservation energy principle, such as increased acceleration and/or heading (Tremoulet \& Feldman, 2000), the C-shaped or S-shaped path of the trajectory (Stewart, 1984; Blythe, Miller, \& Todd, 1996, 1999; Gelman et al., 1995), speed and direction changes (Szego \& Rutherford, 2007; Visch \& Tan, 2009; Träuble, Pauen, \& Poulin-Dubois, 2014), or non-rigid, by rhythmic 
expansion-contraction, motion (Michotte, 1946/1963; Schlottmann \& Ray, 2010), and by mimicking the motion of naturally occurring stimuli (Schultz \& Bülthoff, 2013).

Even if such minimal kinetic variations alone do induce impressions of animacy and goal-directedness, another stream of research has focused on the role of spatiotemporal contingencies between two or more moving objects in generating animacy, as in the Heider \& Simmel's cartoon-like demonstration (1944) and in the Michotte's trigger effect (1946/1963), including more recent works (Bassili, 1976, Dittrich \& Lea, 1994; Schlottmann \& Surian, 1999; Schlottmann et al. 2002; Pavlova, 2012; Schultz, Friston, O'Doherty, Wolpert, \& Frith, 2005). Some authors hypothesized that "displays that contain two or more moving objects appear often more compellingly animate than those depicting only one object, perhaps because each object provides a more rich and variable "context" for the other" (Heberlein 2008 , p. 366). Consequently whenever a simple supporting context like another small geometrical object is added into the same display, animacy ratings significantly increase (Tremoulet \& Feldman, 2006).

In most of these configurations, moreover, beside animacy, viewers tend also to attribute various emotional reactions and intentional states to the moving objects, such as goaldirectedness or chasing, avoiding, fighting, playing and other kinds of social interactions (e.g. Kanizsa \& Vicario, 1968; Asch, 1952; Opfer, 2002; Csibra, 2008; Dasser et al., 1989; Gao, Newman, \& Scholl, 2009; Rochat et al., 2004; Tremoulet \& Feldman, 2006; Gao, McCarthy, \& Scholl, 2010; Gao \& Scholl, 2011; Gao, New, \& Scholl, 2017; Dittrich \& Lea, 1994; Blythe et al., 1999; Barrett et al., 2005; Schlottmann, Ray, Mitchell, \& Demetriou, 2006; Parovel \& Guidi, 2015).

To emphasize the role of relational and contextual cues in the emergence of animacy and mental states, some authors refer to the intentionality hypothesis, which claims that the environment where an action is observed may influence the observer's perception of animacy (Baker et al., 2009; Csibra, 2008; Gao \& Scholl, 2011; for reviews see Tremoulet \& Feldman, 2006; Gao \& Scholl, 2013).

However, there still coexist diverging views in the recent literature. (1) Intentionality itself, that is the appearance of psychological attitudes in the moving object, has been related to goal-directedness, i.e., moving towards something (Gao \& Scholl, 2011; Blythe, Miller \& Todd, 1996), even if the goal is out of the screen (Tremoulet \& Feldman, 2000), (2) while other authors have suggested instead that goal-directedness can be an effective cue in improving animacy, but not intentionality. They define intentionality "in the sense of higherlevel orders of intentionality", that is the presence of mental states (Heberlein, 2008; Opfer, 2002). In the "intentional reaction" (Kanizsa \& Vicario, 1968; see Schlottmann et al., 2006), for instance, a square is described as "seeing" an approaching square and escaping-away from it. Note that, in this case, the object shows a form of awareness even if it is moving away, and not towards, something. Moreover (3), according to other authors, animacy itself depends on intentionality, and so there cannot be animacy without intentionality (Dittrich \& Lea, 1994; Tremoulet and Feldman, 2000), while, according to others (4), animacy and intentionality are separate constructs (Premack, 1990; Gao \& Scholl, 2013). An intermediate position (5) has been proposed by Visch and Tan (2009), stating that animacy could be a basic perceptual property that can be integrated with more complex impressions like emotions and intentions. In this sense, intentionality and goaldirectedness could be conceived as "related properties" of animacy (Gao \& Scholl, 2011) that may be triggered by specific spatiotemporal contexts.

One study specifically centred on the role of context has been reported by Tremoulet and Feldman in 2006. Their displays showed a single figure (rectangular or round) moving on a screen and changing both speed and trajectory simultaneously, while a static object (dot-foil or rectangular paddle) was placed in different positions defining several behavioural conditions of the target, like moving toward a prey, or away from a predator, or being an obstacle. Tremoulet and Feldman found a small but significant effect of the context on animacy ratings, particularly in the goal/prey conditions, and a strong effect of speed change, which replicated previous results from the same group (Tremoulet \& Feldman, 2000). In one of their experiments they also adopted dynamic contexts, to create a temporal contingency between the paddle and the target's change in velocity, but they did not get a significant increment in animacy ratings, even if the goal condition was rated higher than the irrelevant condition. They suggest that a key factor in the perception of animacy is the attribution of an intention to the object, an intention than can be triggered by speed increase and change in direction alone, but that can also be specified by a supporting context, leading the observer to infer the cause of its motion (see Gelman, Durgin \& Kaufman, 1995).

This hypothesis is also supported by a long tradition of research in this topic in experimental phenomenology, according to which we identify causal and social relations on the basis of the kinetic structure of the global configuration, i.e. the way in which two moving objects interact (Michotte, 1946/1963; see Asch, 1952; Bozzi, 1969, 1990; Wagemans, Van Lier, \& Scholl, 2006; Costall, 1991; Thinès, Costall, \& Butterworth, 2015).

The aim of the present research was to explore further the role of different spatiotemporal contexts on the perception of animacy and other related properties - such as emotional states and intentionality - in two-dimensional moving objects. In particular, we focussed on simple basic interactions between the target and a second object acting as a context (similar to Michotte's causal displays), and planned to compare displays where the context is neutral with displays where the nature of the context is specified as an animate agent or as a lifeless object. We expected that both the type of context and 
the relative direction of the target's motion with respect to the same context, i.e. approaching vs avoiding, may influence the observer's perception of animacy and intentionality.

\section{Experiment}

The experiment was designed to investigate if, and how, different kinds of context differ from each other in influencing the impression of animacy elicited by a moving object. The context consisted of a static or moving object, having the same shape of the target object, which could show an animate-like or a physical-like trajectory. ${ }^{1}$ We also contrasted the opposite relative directions between the target object and the contextual object, with the goal to compare approaching vs avoiding displays, keeping unchanged the other stimulus conditions. In simpler words, the contextual object was placed before or after the path of the target object. Finally, we also varied the type of motion of the target on the screen introducing animacy cues in its trajectory by making it move in a way reminiscent of a caterpillar, as did Michotte (1946/1963) in his demonstrations on selfpropelled animal locomotion. In this way we could investigate the relative weight of external (i.e., context) and of intrinsic (i.e., motion type) factors in the perception of animacy.

Most of the studies of perceived animacy reported in the literature used an explicit rating task to measure the perceived animacy. We decided to complement rating tasks with 2alternative forced-choice (2AFC) tasks, in which participants had to choose between two stimuli the one in which the target object seemed more alive. Requiring very spontaneous and intuitive responses, in fact, the forced choice method allows us to obtain results based more on immediate perception than those obtained by a scale that asks for explicit judgements about the impression of animacy (see Parovel \& Guidi, 2015, for another application of this method in a related investigation).

Finally, to determine the effect of different contexts on the emergence of properties related to animacy, such as emotions and intentions, we also recorded the phenomenological reports of participants at the end of the experimental session.

\section{Methods}

\section{Participants}

Participants were 60 university students (42 females) with a mean age of 28 years $(\mathrm{SD}=3.6)$. All participants reported normal or corrected-to-normal vision. All gave their written informed consent in accordance with the Declaration of

\footnotetext{
${ }^{1}$ In contrast to Tremoulet and Feldman, 2006, we used two identical stimuli for target and context, better simulating two interacting agents.
}

Helsinki, and were debriefed at the end of the experiment about the purpose of the study.

\section{Design}

The stimuli were designed starting from two basic configurations in which a small black square, initially static on the screen, briefly moved horizontally at constant speed, either from left to right or vice versa, and then halted (Fig. $1 \mathrm{a}, \mathrm{b})$. Six variations were then devised by introducing a second grey square that provided a context for the motion of the black one. In the static context conditions, the grey square did not move, and it was placed either to the left of the initial position of the black square, so that the latter moved away from it (Fig. 1c), or to the right of the final position of the square at the end its path, so that the black square moved toward the context element (Fig. 1d). In the physical context, the grey square moved downward vertically from an initial position at the top left of the screen or at the top right of it, so that it seemed to fall either next to the starting position of the black square (that thus moved away from it; Fig. 1e), or next to the final position of it (so that the black square moved toward the grey one; Fig. 1f), bouncing twice when it reached the same horizontal position of the black square. Finally, in the animated context conditions, the grey square moved in a way that suggested it was animated, changing its shape during motion, alternating phases of contraction to phases of expansions, thus resembing a caterpillar moving along a linear path. In one of these conditions (Fig. 1g) the grey square seemed to follow the black one, which in turn seemed to move to escape from it. In the other condition (Fig. 1h) the grey square moved toward the black square, which in turn moved toward it, as if it wanted to approach it. Eight further configurations identical to the previous ones were then produced by introducing animacy cues in the motion of the black square, making it move while its shape changed alternating phases of contraction and expansion (examples for the static context condition only are depicted in Fig. 1i-j). Overall 16 stimuli were produced corresponding to all the possible combinations of three factors: type of motion of the black square (two levels), context (four levels) and direction of motion of the square (two levels).

The experiment consisted of 72 trials, divided into three blocks. The first two blocks comprised 28 trials each, corresponding to the all the possible pairwise comparisons of the eight stimuli in which the type of motion of the black square was the same. Approximately half the participants first saw in the first block the stimuli in which the square moved linearly, and the other half first saw the stimuli in which it moved like a caterpillar. In the third block, all 16 stimuli were shown individually, and participants gave free 
a

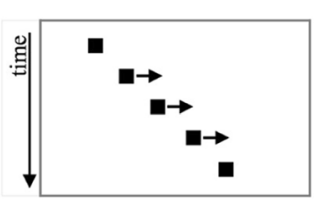

C

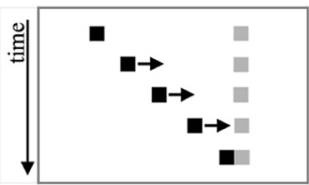

9

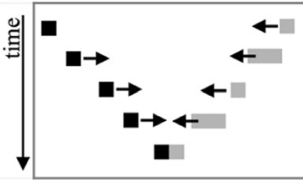

i

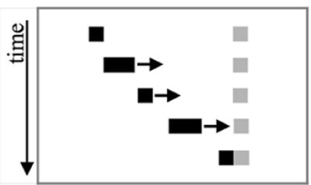

b

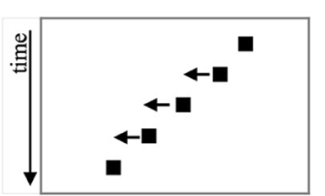

d

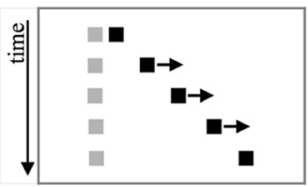

h

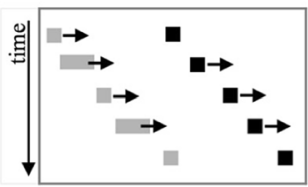

j

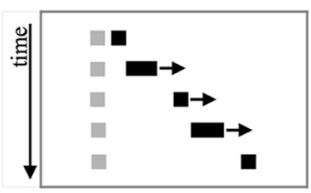

Fig. 1 Schematic depictions of the stimuli used in the experiment. In each panel are represented the relative positions and directions of motion of the target (black square) and of the context element (grey square) at different consecutive time-points during an animation. a, b No context element; $\mathbf{c}-\mathbf{d}, \mathbf{i}-\mathbf{j}$ context square is static; $\mathbf{g}-\mathbf{h}, \mathbf{e}-\mathbf{f}$ context is dynamic, and the context moves, respectively, in an animate fashion

descriptions and rated each of them for the level of animacy displayed by the black square. The order of trials was randomized within each block.

\section{Stimuli}

The stimuli were short animations realized in Adobe Flash Professional CS 5.5 and presented as Quicktime ${ }^{\mathrm{TM}}$ displays. They had variable durations depending on the experimental condition (from $3000 \mathrm{~ms}$ to $4000 \mathrm{~ms}$ ), and consisted in either one or two black and grey squares ( rectangle $\left(\right.$ width $=12.2^{\circ}$, height $=8^{\circ}$ ) placed at the centre of the screen against a blank background (see Fig. 1).

At the onset of the stimuli, the black square was placed on the horizontal midline of the rectangle, at $3^{\circ}$ from its left side in most conditions (in the animated contexts it was placed at $0.75^{\circ}$ from left side of the border, or in the centre of the rectangle, respectively, in the approach and in the escape conditions). Shortly after the onset (from $660 \mathrm{~ms}$ to $1160 \mathrm{~ms}$ after, depending on the condition) the square started to move horizontally toward the left at constant speed $\left(3.72^{\circ} \mathrm{s}\right)$, halting after a displacement of $6.25^{\circ}$. The grey square (i.e., the "context" of the black one) was either absent, static or dynamic, depending on the experimental conditions. When it was dynamic, it started from a $0.75-3^{\circ}$ distance from the white rectangle's left side on the same horizontal line as the black one (and it would then move horizontally on this line),

e

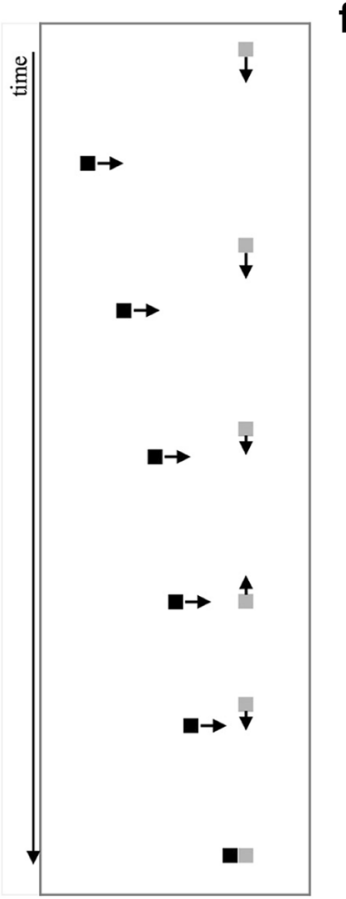

f

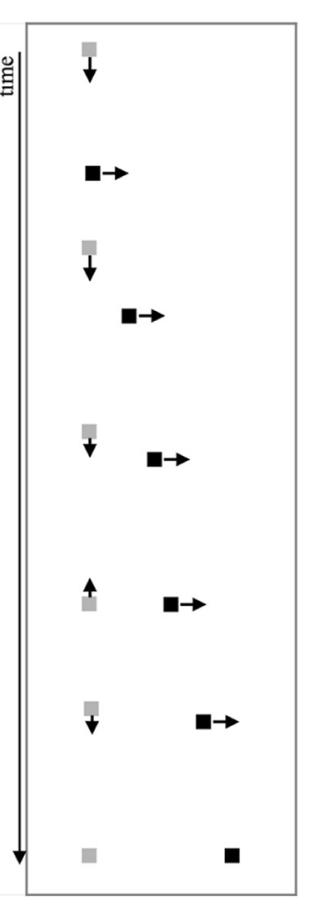

(contracting and expanding while moving) and falling down from above simulating (physical context). In $\mathbf{a}-\mathbf{h}$ the black square moves linearly, while in $\mathbf{i}-\mathbf{j}$ it moves in an animate fashion (contracting and expanding while moving). The full set of displays are available as supplementary material

or from a $0.75^{\circ}$ distance from the rectangle's top (moving then down up to its midline) with a speed of $3-3.72 \%$. Its (final or static) position was always next to the black square's initial or final position (distance $=0.02^{\circ}$ ). At the onset, and at the end of the stimuli, both squares stood still for $660 \mathrm{~ms}$; in the experimental condition in which both moved, the black square started with a $500 \mathrm{~ms}$ delay after the grey one. The full set of displays are available as supplementary materials.

The stimuli were presented on a LED ultrabook monitor, with a screen measuring 13.3 inches diagonally, and participants viewed them from approximately $60 \mathrm{~cm}$, with unrestricted head and eye movements.

\section{Procedure}

The experiment consisted of two parts, the first devoted to 2AFC tasks, and the second to descriptions and ratings.

At the beginning of the first part, participants were instructed about the nature of the task. They were told that, on each trial, they would see two simple animations, one after the other, and that their task was to choose the one in which the black square appeared more animated, i.e., gave the more vivid impression that it were alive. Two blocks of trials were run, each devoted to a type of movement of the black square (i.e., linear or caterpillarlike). At the beginning of each trial, the screen remained blank for $500 \mathrm{~ms}$, and then the first display was played. After the first 
display stopped, the squares were removed and the white rectangle remained empty on the screen for other $1500 \mathrm{~ms}$, before the second display was played. After the second movie stopped, the screen turned blank again, and instructions were shown to the participants: they were told to indicate the movie in which the black square had seemed more animate to them, and to press the left key if they considered the first movie to be more animate, and the right key if they thought the second movie was more animate. After completing a block of trials, participants were given the opportunity to take a break if they desired.

In the second part, participants were shown the displays individually and they were then asked to describe them freely. They were asked to give spontaneous descriptions and to specify, where applicable, the type of interaction between the two elements. The descriptions of participants were recorded on paper by the experimenter to be further analyzed. After eliciting the description of the stimulus, participants were asked to rate the degree of animacy they perceived in the black square, on a 7-point Likert-scale, where " 1 " indicates no impression of animacy, "7" a very clear impression of animacy.

Stimuli presentation and data recording during the first part of the experiment were controlled by PsychoPy2 v1.80.01 software running on an Acer Aspire S3.

\section{Results}

\section{Forced choice data}

Paired comparisons data were analyzed using the BradleyTerry-Luce scaling method (Bradley \& Terry, 1952; Luce, 1959) to derive estimates of the perceived animacy of the target in the different configurations on a ratio scale level. Given that participants only compared in 2AFC tasks stimuli in which the type of motion of the target was the same, separate analyses were conducted to estimate the perceived animacy of the target moving linearly and of the one moving in a caterpillar-like fashion. The validity of the assumptions concerning the consistency of participants' judgements underlying these probabilistic choice models was tested in the following way. We first counted the number of violations of the Weak Stochastic Transitivity assumption, required to place stimuli on an ordinal scale (Tversky, 1969). ${ }^{2}$ No violations of the WST were observed, either in the model for the linear target, or in the one for the caterpillar target, so we did not conduct formal statistical tests. The more stringent assumption of Strong Stochastic Transitivity (SST), ${ }^{3}$ needed to derive estimates on a ratio level

\footnotetext{
$\overline{2}$ Tversky, A. (1969). Intransitivity of preferences. Psychological Review, 76, $31-48$.

${ }^{3}$ If Pxy, the probability of chosing stimulus $x$ over stimulus $y$, and Pyz, the probability of choosing stimulus y over stimulus $\mathrm{z}$ are both $\geq 0.5$, then Weak Stochastic Transitivity implies that Pxz $\geq 0.5$, while Strong Stochasticy Transitivity implies that $\mathrm{Pxz} \geq \max \{\mathrm{Pxy}, \mathrm{Pyz}\}$.
}

scale, was checked by comparing the likelihood of the fitted choice models to the likelihood of the corresponding saturated binomial models that fit the data perfectly, using likelihood ratio tests. In both cases, the tests was not significant [linear movement: $G^{2}(21)=23.2 ; P=0.33$; caterpillar movement: $G^{2}(21)=$ $20.5 ; P=0.49$ ], indicating a good fit of the model, and showing that violations of SST were random rather than systematic, and thus that the model assumption was respected. Finally, we tested for stimulus equality by comparing with likelihood ratio tests the fitted models to models in which the parameters were set as if the probability of any choice among two stimuli was 0.5 . For both types of motion this test was significant, showing that at least two stimuli were considered different in animacy from each other [linear movement: $G^{2}(7)=192.5$; $P<0.0001$; caterpillar movement: $\left.G^{2}(7)=269.9 ; P<0.0001\right]$.

The perceived animacy in the different conditions is plotted in Fig. 2, with error bars representing 95\% confidence intervals. As the plots show, first of all, both in the case of the linear target, and in the one of the caterpillar, the perceived animacy in the control and in the static context conditions was approximately the same, and significantly lower than in the dynamic contexts. Moreover, in the control, and in the static context conditions, there was no difference in animacy as a function of the direction of motion. Conversely, in the dynamic context conditions, the perceived animacy was higher when the target moved away from the contextual element than when it was heading toward it.

Further tests were conducted to assess whether there were differences in the animacy perceived by males and females. Following Duineveld et al. (2000), we compared with likelihood ratio tests, a model in which the parameters (i.e., the scale values) were restricted to be equal across groups to a more complex model that allowed different parameters for each group. Neither in the case of the linear movement of the target $\left[G^{2}(7)=6.28 ; P=\right.$ $0.51]$ nor in the one of the caterpillar movement the test was significant $\left[G^{2}(7)=10 ; P=0.19\right]$, showing that perception of animacy was not different in males and females.

\section{Likert scale ratings}

The ratings of animacy were analysed in a 3-way ANOVA, including type of movement of the target (linear vs caterpillar), context (four levels: no context, static context, physical context, animate context), and direction of motion with respect to the context (toward vs away) as within-subject factors. The results show significant effects of the type of movement $[\mathrm{F}(1,44)=15.49, P<0.001]$ and of the context $[\mathrm{F}(3,132)=$ 26.73; $P<0.001]$. The three-way interaction was also significant $[\mathrm{F}(3,132)=2.95 ; P<0.05]$. No other factors or interactions among factors were significant.

The average animacy ratings are plotted in the top row of Fig. 3, as a function of context (a) and type of movement (b). As can be seen, the pattern of means first of all shows that ratings were higher in the dynamic contexts than in the static 


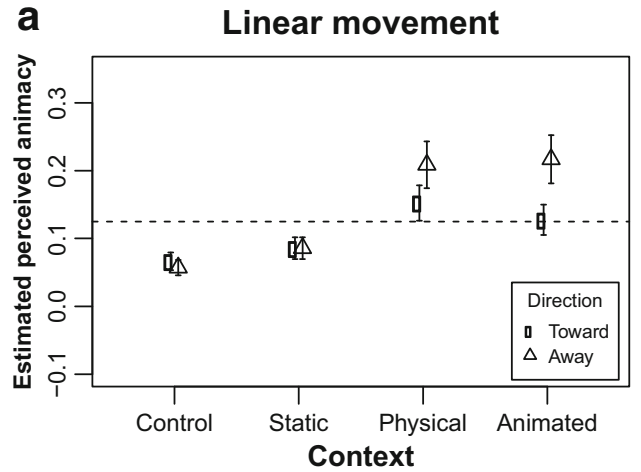

Fig. 2 Perceived animacy scale values estimated from the paired comparison data, as a function of context type and direction of motion. The estimates are normalized so that, for each plot, the values sum to 1 . Values are on a ratio-scale. a Plot of animacy values derived from paired comparisons of stimuli with a linear target. b Corresponding values

one, where, in turn, ratings were higher than in the control nocontext condition, consistent with the results of the paired comparisons. Secondly, the ratings of the animacy of the target were higher when it moved in an animated fashion (caterpillar) than when it moved linearly (difference $=0.64$ ).

We followed up the significant 3-way interaction conducting two sets of separated 2-way ANOVAs, splitting data in one case by type of motion (including context and direction as factors), and in the other by direction of motion (including context and type of motion as factors). In the first
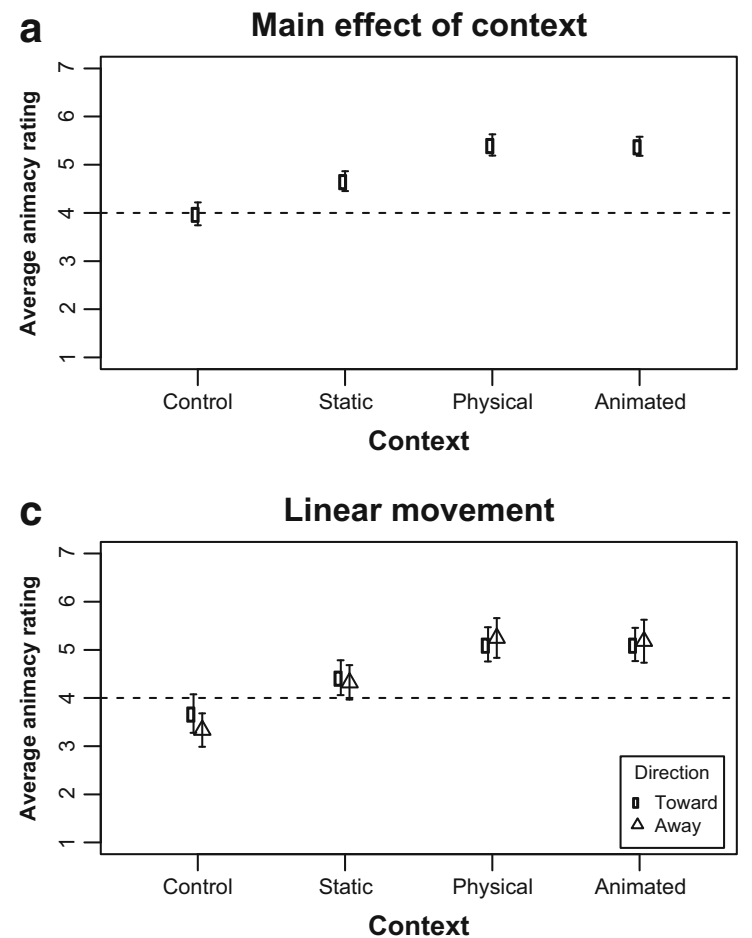

Fig. 3 Plots of the average ratings of the target's animacy in the different conditions. Top row Average ratings by context (a) and type of motion of the target (b). Bottom row 2-way interaction plots of context and type of motion for the target moving away from the context (c) and moving

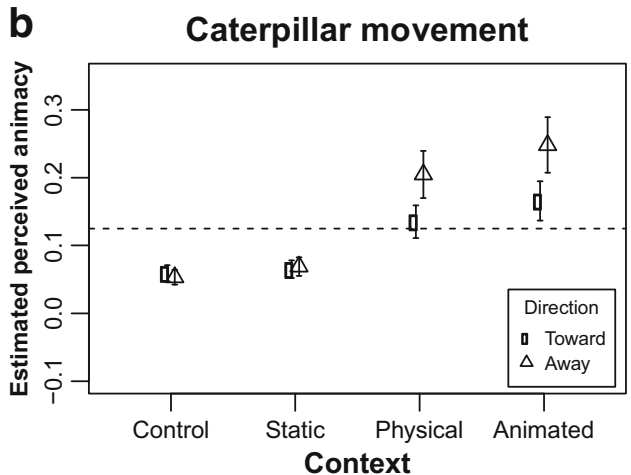

relative to the caterpillar target. Error bars $95 \%$ confidence intervals of the scale values. Dotted line Location on the scale where all the stimuli would lie if, for each pair of stimuli, the probability of choosing one over the other as more animate were 0.5 (i.e., if there were no difference in the perceived animacy)

set of analyses, we always found a significant effect of context only. As shown in Fig. 3 (c-d), in fact, for both types of movement, the direction of motion did not seem to influence the average rating of the target's animacy. The ANOVAs on the data relative to the target moving away from and toward the context square, instead, revealed both significant main effects of context and type of motion and, for the target moving away from the context element, also a significant interaction $[\mathrm{F}(3,132)=3.52 ; \mathrm{p}<.05]$. Further paired sample $t$-tests showed that only in the physical context, and for a target
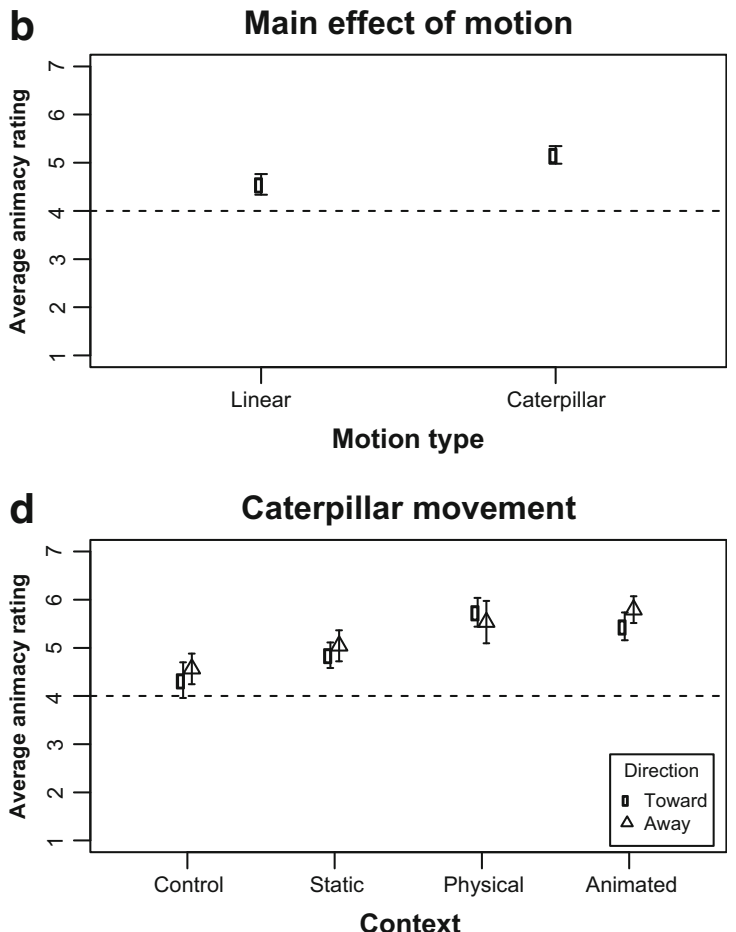

toward it (d). Error bars $95 \%$ confidence intervals of the means adjusted to support inferences about the differences between the means. Dotted line Midpoint of the scale 
moving away from the context, animacy ratings in the caterpillar motion condition were not significantly higher than ratings in the linear motion condition.

\section{Free reports}

To analyse free reports, we first applied thematic coding to each description given by participants of the stimuli. Two ad hoc categorical variables were devised: one (animacy) encoded whether the target was described as an inanimate object or as a living creature, and, in the latter case, also whether the description entailed animacy on an automatic (e.g., "walking"), instinctive (e.g., "flight") or mental level (e.g., a "person", who "wants to reach something"). The second variable encoded instead the emotional valence of the description of the behavior of the target square, on three levels: negative, neutral/no emotion, and positive. We then computed the frequencies of occurrence of the different categories in the overall set of descriptions, and used diverging stacked-frequencies plots to visualize the results. These plots are a variation on the stacked bar chart commonly used to display the frequency of responses in surveys, where each stack represents the frequencies of replies to a question (with replies usually sorted, say from "strongly disagree" to "strongly agree"). In the diverging stacked bar plot version, the replies (i.e., the response categories) are positioned horizontally so that negative responses are stacked to the left of a vertical baseline and positive responses are stacked to the right of this baseline. In case of an odd number of categories, the stacks are centred on the middle category (generally representing a "neutral" response).

Figure $4 \mathrm{a}$ and $\mathrm{b}$ display, respectively, the frequencies of the animacy types and the emotions attributed to the target in the free reports. For each context, type and direction of the target motion, the length (horizontally) of the colored bars represent the percentage of reports in which the target animacy or emotional valence were described using the respective categories. For example, $61 \%$ of participants described the target moving linearly left-to-right in the static context as inanimate, while the remaining 39\% described it as mainly either as "animate, automatic" or "animate, mental". Moreover, 96\% of participants described the valence of the emotion of the target in this condition as "neutral/none", and the remaining $4 \%$ ascribed to the target either a positive $(2 \%)$ or a negative (2\%) emotion. As can be seen, for both the linear target and the caterpillar one the proportion of descriptions of the target as being somehow animated was lowest in the control conditions, and highest in the dynamic context conditions. In the control condition, the proportions of description of the target as being animate were higher when it moved like a caterpillar $(70 \%)$ than when its movement was linear (39-44\%). This was also true in the static context, although the proportions of animacy attributions were higher than in the control condition, and substantially above $50 \%$ for both types of targets. In the dynamic context, only a small percentage of participants judged the target to be inanimate $(7-12 \%)$; moreover, the majority of subjects described the target's animacy as either instinctive or mental. Interestingly, the proportions of descriptions of the target as being driven by mental states was higher when it moved toward the context square than when it moved away from it.

Looking at the plots of the emotions attributed to the target behaviour, we can notice first of all that, in the control condition (no context), almost all participants did not attribute an emotional state to the target. Secondly, in the other contexts participants tended to attribute a negative emotion to the target when it was moving away from the context, and a positive one when it was approaching it. The percentage of participants describing the target with reference to an emotional state, finally, was highest in the dynamic context when the target was moving toward the context square.

\section{Discussion and conclusions}

The main results show that the attribution of animacy is significantly influenced by the features of the context where the target object is moving. Even in presence of clear cues of biological motion, that is when the target object moves in a "caterpillar-like" manner, there do still exist different levels of perceived animacy on the basis of different contexts, instead of an even distribution of the levels of perceived animacy, as we would expect if animacy were an all-or-none property. To vary the context we adopted a simple stimulus identical to the target, located at the beginning or at the end of its trajectory, in static or dynamic conditions (physical or animate).

Our main results indicate that, in both the forced-choice and the Likert-scale tasks, there is a significant difference between static and dynamic contexts, where the dynamic contexts prompt a quite clearer impression of animacy, while for the physical and the animate contexts there are similar results in almost all conditions. We also found a significant difference in the impression of animacy between the control situation without context and all the other conditions.

As a possible explanation of this first finding, we suggest that, independently from the specificity of the relationship between the target object and the context object, a dynamic context produces a spatiotemporal contingency comparable to Michotte's paradigm of perceptual causality. Like in the launching paradigm, in fact, the target object starts to move as a reaction to the behaviour of another object, either moving closer/escaping or bouncing nearby. On the other side, the static context object could be inferred as being the cause of the target object's motion, but without temporal constraints this causal impression is much less vivid and compelling. 
a

Animacy type attributed to target

1: Control, left-to-right

\begin{tabular}{r|lrr} 
Linear & $61 \%$ & & $39 \%$ \\
Caterpillar & $30 \%$ & & $70 \%$ \\
\cline { 3 - 4 } & & 2: Control, right-to-left & \\
Linear & $56 \%$ & & $44 \%$ \\
Caterpillar & $30 \%$ & & $70 \%$
\end{tabular}

3: Static Context, away

\begin{tabular}{r|llr|} 
Linear & $28 \%$ & & $72 \%$ \\
Caterpillar & $11 \%$ & & \\
& & & \\
& & 4 4: Static Context, toward \\
Linear & $32 \%$ & & $68 \%$ \\
Caterpillar & $14 \%$ & & $86 \%$
\end{tabular}

\begin{tabular}{r|rrr} 
Linear & $12 \%$ & & $88 \%$ \\
Caterpillar & $9 \%$ & & $91 \%$ \\
\hline
\end{tabular}

6: Physical Context, toward

\begin{tabular}{r|lrr}
\cline { 2 - 3 } Linear & $16 \%$ & & $84 \%$ \\
Caterpillar & $14 \%$ & & $86 \%$ \\
& & &
\end{tabular}

\begin{tabular}{r|ccr} 
& \multicolumn{3}{c}{ 7: Animate Context, away } \\
\cline { 2 - 3 } Linear & $11 \%$ & & $89 \%$ \\
Caterpillar & $9 \%$ & $91 \%$
\end{tabular}

8: Animate Context, toward

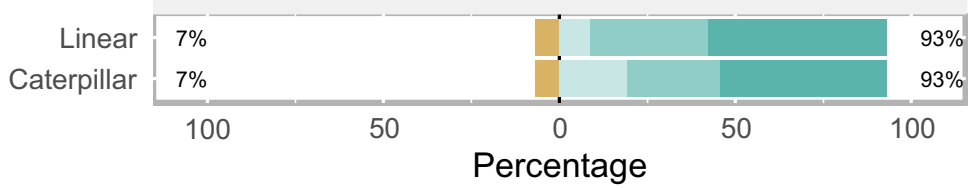

Type: inanimate animate animate animate

\section{b Valence of emotion of target}

1: Control, left-to-right

\begin{tabular}{|c|c|c|}
\hline $2 \%$ & $96 \%$ & $2 \%$ \\
$5 \%$ & $89 \%$ & $5 \%$ \\
\hline & 2 2: Control, right-to-left & \\
\hline $11 \%$ & $86 \%$ & $4 \%$ \\
$7 \%$ & $91 \%$ & $2 \%$ \\
\hline
\end{tabular}

3: Static Context, away

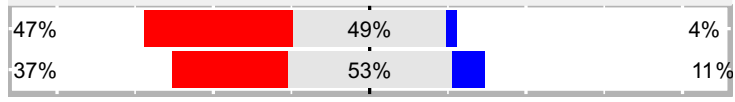

4: Static Context, toward

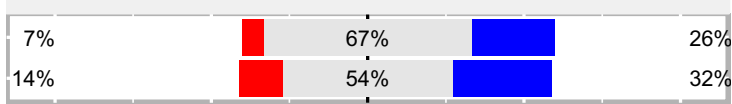

5: Physical Context, away

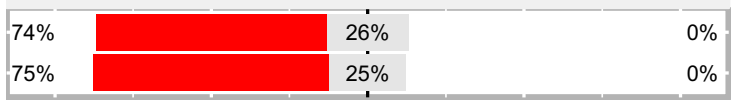

6: Physical Context, toward

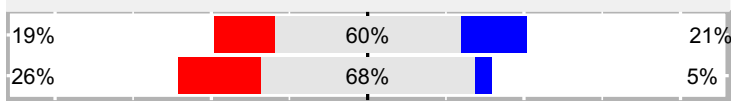

7: Animate Context, away

\begin{tabular}{|c|c|c|}
\hline $74 \%$ & $26 \%$ & $0 \%$ \\
\hline $60 \%$ & $33 \%$ & $7 \%$ \\
\hline
\end{tabular}

8: Animate Context, toward

\begin{tabular}{|c|c|c|c|c|}
\hline $18 \%$ & & $35 \%$ & & $47 \%$ \\
\hline $2 \%$ & & $53 \%$ & & $46 \%$ \\
\hline 100 & 50 & $\begin{array}{c}0 \\
\text { cent }\end{array}$ & 50 & 100 \\
\hline
\end{tabular}

Fig. 4 Diverging stacked bar plots of the frequency of (a) the types of animacy attributed to the target, and (b) the emotions ascribed to its behavior in the free descriptions of the stimuli, as function of the type of movement of the target (Linear vs Caterpillar). For each context, type, and direction of the target motion, the length (horizontally) of the colored bars represent the frequency (percentage) of occurrence of the various categories for the target animacy (sand/left inanimate, green/right animate, shades of green types of animacy) and the emotional valence (red/left negative, grey/middle no emotion or neutral, blue positive) in the free reports. Each stack of bars has a total length of $100 \%$. The side toward which the stack is displaced with respect to the chosen baseline or the central category reflect the prevalence (i.e., higher frequency) of categories of a given polarity over the other. The frequency of each polarity is presented in the margins, as annotations at the ends of the scale. See text for further details
A second meaningful new finding emerged from the paired-comparisons data, concerning the role of the direction of the target's motion with respect to the context, while in the control and in the static context condition, there were no differences in the perceived animacy of the target as a function of its direction of motion, in the dynamic contexts we consistently found that the impression of animacy was higher when the target was moving away from the context element than when it was approaching it.
This effect was not found in the ratings data. Possibly this discrepancy is due to the lower sensitivity of the method relative to paired-comparisons, which, forcing the viewer to make a choice, allows the identification of even small differences that would otherwise pass unnoticed. Thus, it supports the effectiveness of adopting the forced-choice method, which avoids enquiring about an explicit quantitative judgement from the participants, in measuring more qualitative and subtle impressions of motion. Besides corresponding to the other measures based on 
the forced-choice task, the Likert ratings show that the displays chosen as animate in the forced-choice really seem animate, by themselves and not only in relation to each other. We must, however, note that neither the paired comparison data nor the ratings allow us to pinpoint the level of the processes behind our results, and thus to tell whether they are more due to automatic low level perceptual processes or to higher-level conscious inferential cognitive processes. However, at least the finding that perceived animacy was equally low in absence of context and in a static one, does not seem to be easily explained by inferential processes, as the static context displaying a goal-directed motion would seem to support inferences about intentionality and animacy that would not be possible without context.

For a better understanding of the results, we referred also to the phenomenal reports of the participants. First, spontaneous descriptions show that the increased animacy in the dynamic contexts corresponds to a parallel increasing of the use of terms generally related to animate behaviors. Within this category, moreover, participants freely described the moving target in very different ways, specifically related to the different contexts. This revealed a range of animate impressions that, together with the aforementioned results that animacy is perceived more or less clearly in different conditions, according to us confirms the hypothesis that there can be different facets or "levels" inside the animacy concept - for instance, an automatic animacy, an instinctive one and a mental/emotional oneand that the context plays a crucial role in making them evident.

Animacy, according to us, can be conceived as an expressive quality of motion, which is articulated in more general characteristics, like simple aliveness, and more specific ones, like intentional or emotional aspects. The overall results corroborate the theoretical assumption according to which intentionality and other emotions are "related properties" of animacy (Visch \& Tan 2009; Gao \& Scholl, 2011).

Secondly, the relative direction of the target motion strongly influenced the free descriptions of the participants. The "moving away" condition is related to negative emotions, while the "approaching" condition generally evokes positive emotions, and, in general, the avoiding behavior is more often described using emotional terms. In addition, comparing the descriptions of the animate motion, it is more "instinctive", i.e., reflexive an animal-like behavior, in the "moving away" condition, and more mentally driven in the "approaching" condition.

As we can see, for some aspects the qualitative reports can be enlightening in suggesting some possible explanations of the data. The "moving away" from something or someone may look more animated for evolutionary reasons, because of the more adaptive meaning of escaping, or for a general predisposition to note emotionally negative events. The negativity bias, known in different areas of cognition and attention, in fact, predicts a higher sensitivity to negative events. Therefore, both "real" and schematic-geometrical events with a negative content or outcome, like, e.g., chasing, according to Heberlein
(2008) are easily perceived as more intentional. Also, with Johansson's dynamic of point-light displays, anger and fear are easier to identify than other emotions (Walk, 1984). Nevertheless, further studies and the development of more objective paradigms in this field of research will be necessary to corroborate this hypothesis, and explore how these findings can be generalized and applied in different contexts.

Phenomenal descriptions support also a relationship between the attribution of intentionality (i.e., the presence of mental states) and the direction toward something/someone, that is goal-directedness (Gao \& Scholl, 2011; Blythe, Miller \& Todd, 1996; Tremoulet \& Feldman, 2000), and an independency between animacy and intentionality, as some authors have claimed (Premack, 1990; Gao \& Scholl, 2013). Also, neural recordings recently gave strong support to the independent constructs hypothesis, showing that the areas involved in animacy/ goal-directedness perception on one side and intentionality on the other [i.e., temporoparietal cortex, including areas in and near the posterior superior temporal sulcus (pSTS) and angular gyrus, particularly in the right hemisphere] are topically the same but manifest different degrees of activation with different patterns (Gao, Scholl \& McCarthy, 2012), leading to a functional distinction among these concepts, even if they must be strictly interconnected (see Heberlein, 2008).

In conclusion, we suggest that at least two main different factors seem to be effective in the perception of animacy and intentionality. On one side, at the same conditions, as the reviewed literature showed, kinematic variables such as sudden speed accelerations and high values of speed, play a crucial role in evoking and intensifying the impression of animacy (see Tremoulet \& Feldman, 2000, 2006). On the other side, it seems very fruitful to deeply investigate the role of spatiotemporal context in eliciting animacy and other related properties, like intentionality and emotional or social behaviours. As happens with other displays of phenomenal causality like triggering and intentional reaction (Michotte, 1946/1963; Kanizsa \& Vicario, 1968; Bozzi, 1969; Schlottmann et al., 2006; Thinès et al, 1991; Parovel \& Casco, 2006; Parovel, 2012; Hubbard, 2013a, 2013b; Parovel \& Guidi, 2015; Wagemans et al., 2006), the perceptual belongingness to a unitary spatiotemporal structure specifies the expressive meaning of the trajectory and the direction of the target motion, and can plausibly influence in this way the perceptual saliency of animacy, also on the basis of the adaptive value of the behavior.

According to an evolutionary-biological account, in fact, all emotions derive from fundamental actions present in the most primitive organisms, which can have two main types of causes: approach (to partner or food) and avoidance (of predators) (Kandel, 2011). Because an effortless and automatic discrimination between animate and inanimate is crucial to successfully avoid a dangerous agent or to approach prey, it should necessarily be an adaptive advantage not only to 
recognize life, but also to discriminate the specific intentions of the agent (Scholl \& Gao, 2013; Neri, Luu, \& Levi, 2006).

And it is the relationship occurring between two or more moving objects, to conclude with Michotte's words, that specifies the meaning of an object's motion, mainly on the basis of spatial and temporal contingencies (Michotte, 1950; Thinès, Costall, \& Butterworth, 2015).

Acknowledgements We are very grateful to Alan Costall and to an anonymous reviewer for their helpful comments on improving this paper.

\section{References}

Asch, S.E. (1952). Social psychology. Englewood Cliffs, NJ: PrenticeHall.

Baker, C., Saxe, R., \& Tenenbaum, J. (2009). Action understanding as inverse planning. Cognition, 113, 329-349.

Barrett, H.C., Todd, P.M., Miller, G.F. \& Blythe, P.W. (2005). Accurate judgements of intention from motion cues alone: a cross-cultural study. Evolution and Human Behavior 26, 313-331.

Bassili, J.N. (1976). Temporal and spatial contingencies in the perception of social events. Journal of Personality and Social Psychology 33(6), 680-685.

Blythe, P.W., Miller, G.F. \& Todd, P.M. (1996). Human simulation of adaptive behavior: Interactive studies of pursuit, evasion, courtship, fighting, and play. In: P. Maes, M.J. Mataric, J.-A. Meyer, J. Pollack \& S.W. Wilson, From animals to animats 4: Proceedings of the Fourth International Conference on Simulation of Adaptive Behavior, 13-22.

Blythe, P., Miller, G. F., \& Todd, P. M. (1999). How motion reveals intention: categorizing social interactions. In: G. Gigerenzer, P. M. Todd, and ABC Research Group (Eds), Simple Heuristics That Make us Smart (pp. 257-285), New York, NY: Oxford University Press.

Bozzi, P. (1969). Unità, identità, causalità. Bologna: Cappelli.

Bozzi, P. (1990). Fisica Ingenua. Milano: Garzanti.

Bradley, R.A. \& Terry, M.E. (1952). Rank analysis of incomplete block designs: I. The method of paired comparisons. Biometrika, 39, 324 345 .

Costall, A. (1991). The background to Michotte's experimental phenomenology. In: G. Thinès, A. Costall, G. Butterworth (Eds), Michotte's Experimental Phenomenology of Perception (pp. 3-12), Hillsdale, New Jersey: Erlbaum.

Csibra, G. (2008). Goal attribution to inanimate agents by 6.5-month-old infants. Cognition, 107, 705-717.

Dasser, V., Ulbaek, I., \& Premack, D. (1989). The perception of intention. Science 243, 365-367.

Dittrich W.H. \& Lea, S.E.G. (1994). Visual perception of intentional motion. Perception 23(3), 253-268.

Duineveld, C.A.A., Arents, P., \& King, B.M. (2000). Log-linear modelling of paired comparison data from consumer tests. Food Quality and Preference, 11, 63-70.

Gao, T., McCarthy, G. \& Scholl, B.J. (2010). The wolfpack effect: Perception of animacy irresistibly influences interactive behavior. Psychological Science 21, 1845-1853.

Gao, T., New, J.J. \& Scholl, B.J. (2017). The wavering wolf: Perceived intentionality controls attentive tracking. Available at: http://www. yale.edu/perception/Brian/demos/animacy-ChasingAttention.html.

Gao, T., Newman, G. \& Scholl, B. J. (2009). The psychophysics of chasing: a case study in perception of animacy. Cognitive psychology 59(2), 154-197.
Gao, T. \& Scholl, B. J. (2011). Chasing vs. Stalking: interrupting the perception of animacy. Journal of Experimental Psychology: Human Perception and Performance 37(3), 669-684.

Gao, T., Scholl, B.J. \& McCarthy, G. (2012). Dissociating the detection of intentionality from animacy in the right posterior superior temporal sulcus. Journal of Neuroscience The Journal of Neuroscience 32(41), 14276-14280.

Gelman, R., Durgin, F. \& Kaufman, L. (1995). Distinguishing between animates and inanimates: not by motion alone. In D. Sperber, D. Premack, and A. J. Premack (cur.): Causal cognition: A multidisciplinary debate (pp. 151-184). New York: Clarendon.

Heberlein, A. (2008). Animacy and intention in the brain: neuroscience of social event perception. In Shipley, T.F., Zacks, J.M. (Eds.): Understanding Events. From Perception to Action (pp. 363-388). Oxford: Oxford University Press.

Heider, F., \& Simmel, M. (1944). An experimental study of apparent behavior. American Journal of Psychology 57, 243-259.

Hubbard, T.L. (2013a). Phenomenal causality I: Varieties and variables. Axiomathes, 23, 1-42.

Hubbard, T.L. (2013b). Phenomenal causality II: Integration and implication. Axiomathes, 23, 485-524.

Kandel, E.R. (2011). The Age of insight: the quest to understand the unconscious in art, mind, and brain: from Vienna 1900 to the present. New York: Random House.

Kanizsa G., \& Vicario G. (1968). The perception of intentional reaction. In G. Kanizsa \& G. Vicario (Eds.), Experimental research in perception (pp. 71-126). Trieste, Italy: University of Trieste.

Luce, R.D. (1959). Individual choice behavior: a theoretical analysis. New York: Wiley.

Markson, L., \& Spelke, E. (2006). Infants' rapid learning about selfpropelled objects. Infancy 9, 45-71.

Michotte, A. (1946/English transl. 1963). The Perception of Causality. London: Methuen.

Michotte, A. (1950). The emotions regarded as functional connections. In M.L. Reymert (Ed.), Feelings and emotions: the Mooseheart Symposium (pp. 114-126). New York: McGraw-Hill.

Neri, P., Luu, J.Y., \& Levi, D.M (2006). Meaningful interactions can enhance visual discrimination of human agents. Nature Neuroscience 9(2), 1186-1192.

Opfer, J.E. (2002). Identifying living and sentient kinds from dynamic information: the case of goal-directed versus aimless autonomous movement in conceptual change. Cognition 86(2), 97-122.

Pavlova, M.A. (2012). Biological motion processing as a hallmark of social cognition. Cerebral Cortex, 22, 981-995.

Parovel G., \& Casco C. (2006). The psychophysical law of speed estimation in Michotte's causal events. Vision Research, 46, 4134-4142.

Parovel, G. (2012). Le qualità espressive. Fenomenologia sperimentale e percezione visiva. Milan: Mimesis.

Parovel, G. \& Guidi, S. (2015). The psychophysics of comic: effects of incongruity in causality and animacy. Acta Psychologica 159, 22-32.

Premack, D. (1990). The infant's theory of self-propelled objects. Cognition 36(1), 1-16.

Rochat, P., Striano, T., \& Morgan, R. (2004). Who is doing what to whom? Young infants' developing sense of social causality in animated displays. Perception, 33, 355-369.

Schlottmann, A., Allen, D., Linderoth, C. \& Hesketh, S. (2002). Perceptual causality in children. Child Development 73(6), 16561677.

Schlottmann, A., Ray, E.D., Mitchell, A. \& Demetriou, N. (2006). Perceived physical and social causality in animated motions: spontaneous reports and ratings. Acta Psychologica 123, 112-143.

Schlottmann, A., \& Ray, E. (2010). Goal attribution to schematic animals: do 6-month-olds perceive biological motion as animate? Dev. Sci. $13,1-10$.

Schlottmann, A. \& Surian, L. (1999). Do 9-month-olds perceive causation-at-a-distance? Perception 28(9), 1105-1113. 
Scholl, B.J. \& Gao, T. (2013): Perceiving animacy and intentionality: visual processing or higher-level judgment? In M.D. Rutherford \& V.A. Kuhlmeier (Eds.), Social perception: detection and interpretation of animacy, agency, and intention (pp. 197-230). Cambridge, MA: MIT Press.

Scholl, B.J. \& Tremoulet, P.D (2000): Perceptual causality and animacy. Trends in Cognitive Sciences 4(8), 299-309.

Schultz, J., Friston, K. J., O’Doherty, J., Wolpert, D. M., \& Frith, C. D. (2005). Activation in posterior superior temporal sulcus parallels parameter inducing the percept of animacy. Neuron, 45(4), 625-635.

Schultz, J. \& Bülthoff, H.H. (2013). Parametric animacy percept evoked by a single moving dot mimicking natural stimuli. Journal of Vision 13(4), 15.

Stewart, J. A. (1984). Object motion and the perception of animacy. Paper presented at the meetings of the Psychonomic Society, San Antonio, TX.

Szego, P.A. \& Rutherford, M.D. (2007). Actual and illusory differences in constant speed influence the perception of animacy similarly. Journal of Vision, 7(12), 5 .

Thinès, G., Costall, A., \& Butterworth, G.E. (Eds.) (2015). Michotte's experimental phenomenology of perception. Hillsdale, NJ: Erlbaum.
Träuble, B., Pauen, P. \& Poulin-Dubois, D. (2014). Speed and direction changes induce the perception of animacy in 7-month-old infants. Frontiers in Developmental Psychology 5. Available at: http:// journal.frontiersin.org/Journal/10.3389/fpsyg.2014.01141/full.

Tremoulet, P.D. \& Feldman, J. (2000). Perception of animacy from the motion of a single object. Perception 29, 943-951.

Tremoulet, P.D. \& Feldman, J. (2006). The influence of spatial context and the role of intentionality in the interpretation of animacy from motion. Perception \& Psychophysics 68(6), 1047-1058.

Tversky, A. (1969). Intransitivity of preferences. Psychological Review, $76,31-48$.

Visch, V.T. \& Tan, E.S. (2009). Categorizing moving objects into film genres: the effect of animacy attribution, emotional response, and the deviation from non-fiction. Cognition 110(2), 265-272.

Wagemans, J., Van Lier, R., \& Scholl, B. J. (2006). Introduction to Michotte's heritage in perception and cognition research. Acta Psychologica, 123, 1-19.

Walk, R.D. (1984). Event perception, perceptual organization and emotion. In W.R. Crozier \& A. J. Chapman (Eds.), Cognitive Processes in the perception of art (pp. 211-221). Amsterdam: North-Holland. 